\title{
Systematic Modal Analysis of 3-D Dielectric Waveguides Using Conventional and High Accuracy Nonstandard FDTD Algorithms
}

\author{
Kosmas L. Tsakmakidis, Christian Hermann, Andreas Klaedtke, Cécile Jamois, and Ortwin Hess
}

\begin{abstract}
A robust methodology is presented for the accurate modal analysis of three-dimensional dielectric waveguides with the finite-difference time-domain (FDTD) method. We investigate the propagation of well-defined vector modes along strongly guiding rectangular waveguides. Eigensolutions of the vectorial wave equation are utilized in the simulations to accurately launch the fundamental and higher order eigenmodes. Results for their FDTD-computed propagation constant are found to be in excellent agreement with existing mode-solving techniques. Improved accuracy or significant computational savings are achieved when the nonstandard FDTD concepts are incorporated in the context of the present analysis.
\end{abstract}

Index Terms-Finite-difference time-domain (FDTD) method, modeling, nonstandard finite-difference (NSFD), optical waveguide.

\section{INTRODUCTION}

$\mathbf{I}$ $\mathrm{N}$ THE design and performance assessment of modern photonic devices, popular numerical techniques, such as the beam propagation method (BPM) or the coupled-mode theory (CMT), are often rendered insufficient. A viable modeling option then can be the three-dimensional (3-D) full-wave finite-difference time-domain (FDTD) method based directly on Maxwell's equations. Typical examples of such applications include the investigation of the in- and out-coupling of a strongly guiding rectangular waveguide to a planar photonic crystal (PPC) [1] or the study of several travelling-wave (TW) devices such as photodetectors, modulators, and phototransistors [2]. The high index contrast in the planar heterostructure and the wide-angle scattering at the horizontal plane of the PPC can render the use of the BPM problematic. The CMT will be ineffective as well since it usually considers the propagation of the first two guided modes and neglects the coupling to the radiation modes [3].

In the FDTD analyses of both classes of applications, the precision in the excitation of specific eigenmodes inside the 3-D integrated waveguide is of crucial importance. The absence of exact solutions usually leads to the employment of less accurate, semianalytical techniques, such as the effective-index method, for the provision of the two-dimensional (2-D) excitation profile, which in practice results in the contamination of the simulation outcomes with unwanted modes. As a result, for the 3-D

Manuscript received March 2, 2005; revised August 16, 2005.

The authors are with the Advanced Technology Institute, School of Electronics and Physical Sciences, University of Surrey, Guildford GU2 7XH, UK (e-mail: K.Tsakmakidis@surrey.ac.uk).

Digital Object Identifier 10.1109/LPT.2005.859174
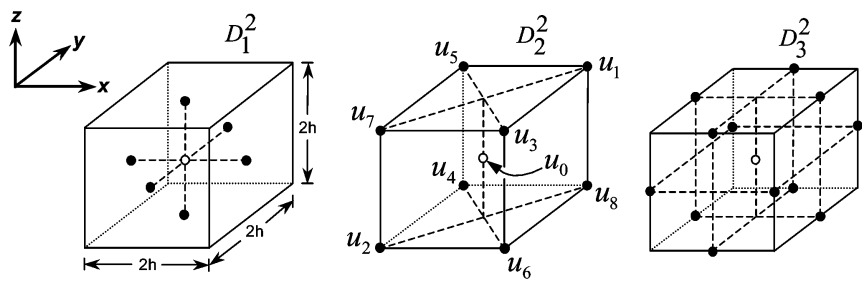

(a)
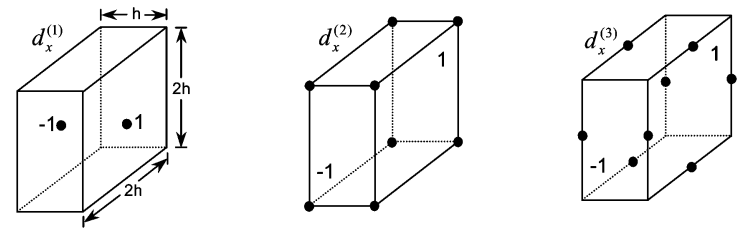

(b)

Fig. 1. (a) Spatial arrangement of the generalized 3-D Laplacian operators. (b) The elements of the nonstandard difference operator $\tilde{d}_{x}$. A suitable weighting component is shown for the filled points in each side of the element.

FDTD modeling of several photonic devices that involve rectangular waveguides, there is currently a need for a robust and general methodology to excite specific eigenmodes.

The purpose of this letter is dual: First, we incorporate an accurate fully vectorial wave equation-based excitation scheme in the 3-D FDTD simulations that manages to launch accurately the desired electromagnetic eigenmodes inside the planar waveguiding heterostructure. The precision in the mode excitation, however, is not sufficient to provide accurate simulation outcomes, particularly for problems that involve propagation over long distances, owing to the cumulative nature of the induced numerical dispersion errors. To overcome that, a highly accurate nonstandard FDTD (NS-FDTD) strategy is adopted which proved to require significantly less computational resources compared to the classical Yee algorithm for the same level of accuracy, as discussed in Section II.

\section{NS-FDTD SimULATION OF THE 3-D DIELECTRIC WAVEGUIDE}

Critical for the NS-FDTD formulae [4], [5] is the introduction of generalized 3-D Laplacian operators, $D_{i}^{2}(i=1, \ldots, 3)$, shown in Fig. 1(a). Focusing on the derivation of $D_{2}^{2}$, we start by introducing the parameters: $\hat{\xi}=h(\partial / \partial x), \hat{\eta}=h(\partial / \partial y)$, and $\hat{\mu}=h(\partial / \partial z)$, where $h$ is a real number representing the cell size and

$$
\left(\hat{\xi}^{2}+\hat{\eta}^{2}+\hat{\mu}^{2}\right) u(\bar{r})=h^{2} \nabla^{2} u(\bar{r})
$$


with $u(\bar{r})$ being an electromagnetic field component in 3-D space. Denoting with $u_{i}(i=1, \ldots, 8)$ the values of the function $u(\bar{r})$ at the points shown in Fig. 1(a), we have

$$
\begin{aligned}
u_{1} & =e^{(\hat{\xi}+\hat{\eta}+\hat{\mu})} u_{0} \\
& =\left[1+(\hat{\xi}+\hat{\eta}+\hat{\mu})+\frac{(\hat{\xi}+\hat{\eta}+\hat{\mu})^{2}}{2}+\cdots\right] u_{0} .
\end{aligned}
$$

Similarly: $u_{2}=e^{(-\hat{\xi}-\hat{\eta}-\hat{\mu})} u_{0}, u_{3}=e^{(\hat{\xi}-\hat{\eta}+\hat{\mu})} u_{0}$, with analogous expressions for the remaining points. Using series expansions like the one in (2) and the identity in (1), we obtain

$$
S_{2}=\sum_{i=1}^{8} u_{i}=8 u_{0}+4 h^{2} \nabla^{2} u_{0}+0\left[h^{4}\right]
$$

from which we can define the second-order Laplacian $D_{2}^{2}$ as

$$
D_{2}^{2} u_{0}=\frac{S_{2} / 4-2 u_{0}}{h^{2}} .
$$

Likewise, the second-order operators $D_{1}^{2}$ and $D_{3}^{2}$ of Fig. 1(a) can be derived, as well as higher order Laplacians. We can efficiently combine $D_{i}^{2}(i=1, \ldots, 3)$ to create an optimal Laplacian operator $D_{0}^{2}$ which equals a suitably weighted sum of $D_{i}^{2}$ and is sixth-order accurate in space for a reference frequency [4]. Decomposing $D_{0}^{2}$ into finite-difference operator products and employing nonstandard stipulations, yields the following spatial formulae that preserves the $0\left[h^{6}\right]$ accuracy [Fig. 1(b)]:

$$
\tilde{d}_{x}=\frac{1}{S_{k}(h)}\left(\hat{\zeta}_{1} d_{x}^{(1)}+\hat{\zeta}_{2} d_{x}^{(2)}+\hat{\zeta}_{3} d_{x}^{(3)}\right)
$$

with $S_{k}(h)=2 \sin (\mathbf{k} h / 2) / \mathbf{k}$ [4], $\mathbf{k}$ being the wavevector that corresponds to the central frequency of the input source and $\zeta_{i}(i=1, \ldots, 3)$ positive weighting components fulfilling $\zeta_{1}+$ $\zeta_{2}+\zeta_{3}=1$ for numerical consistency. For the other two directions analogous difference operators can be formed which, when inserted into the $\boldsymbol{E}$ or $\boldsymbol{H}$-field updating equations, result in a significantly ameliorated FDTD scheme with regard to numerical dispersion and anisotropy

Very crucial for the simulations herein is the formulation of the nonstandard uniaxial perfectly matched layer inside which extend the waveguide layers. For the sake of brevity, only the expression for the advancement of the $B_{y}$ component (in Heaviside- Lorentz units) is given below

$$
\begin{aligned}
B_{y}=\frac{\kappa_{z}-\frac{S_{\omega}(\Delta t) \sigma_{x}}{\left(2 \varepsilon_{0}\right)}}{\kappa_{z}+\frac{S_{\omega}(\Delta t) \sigma_{x}}{\left(2 \varepsilon_{0}\right)}} B_{y} \\
\quad+c_{0} \frac{S_{\omega}(\Delta t)}{\kappa_{z}+\frac{S_{\omega}(\Delta t) \sigma_{x}}{\left(2 \varepsilon_{0}\right)}}\left(\tilde{d}_{x} E_{z}-\tilde{d}_{z} E_{x}\right)
\end{aligned}
$$

with $S_{\omega}(\Delta T)=2 \sin (\omega \Delta t / 2) / \omega, c_{0}$ the velocity of light in vacuum, and both finite-difference operators, now involving 18 instead of two points of a typical formulation [6].

For the acquisition of the 2-D excitation profiles, we note that in rectangular dielectric waveguides, there are no precise closed-

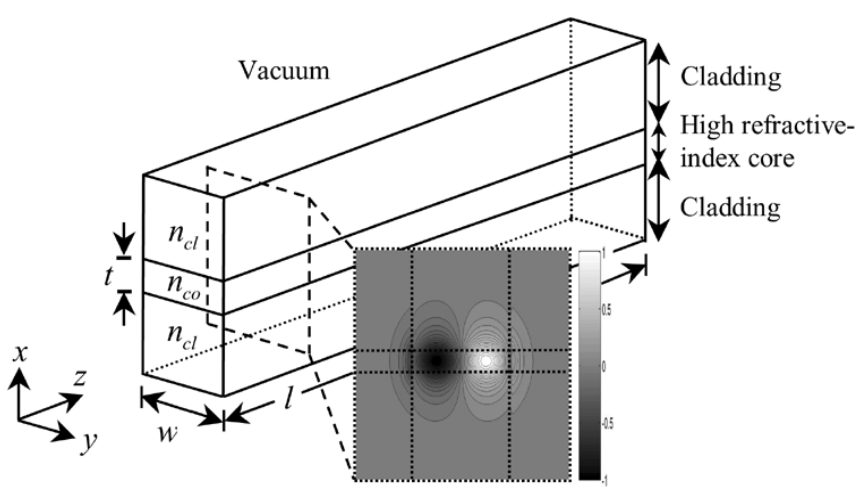

Fig. 2. Strongly guiding planar heterostructure coupled to a PPC line-defect waveguide. Also shown is the precalculated excitation profile for the generation of the $E_{12}^{y}$ mode inside the rectangular waveguide.

form expressions for the cross-sectional shape of the modes, owing to the peculiar behavior of the electromagnetic fields near the dielectric corners where they diverge to a small degree [7]. A sufficiently accurate numerical way to overcome this limitation is via computing the $\boldsymbol{E}$ - or $\boldsymbol{H}$-field eigenvectors of the vectorial wave equation that takes into account the polarization, vector properties, and discontinuity of the guided modes at the dielectric interfaces, including the corner regions [8]. Upon obtaining the 2-D field distributions in this manner, a proper spatial interpolation is necessary to match the Yee grid. Such an approach is essential, particularly for FDTD in-coupling studies, and an exemplary result for the dominant component of the $E_{12}^{y}$ eigenmode is illustrated in Fig. 2. The associated propagation constant is obtained from the eigenvalue of the wave equation and, for sufficiently small mesh size, the error in its estimation can be made very small (e.g., less than $0.01 \%$ ) [8].

The structure on which the overall methodology was tested is a multimode rectangular waveguide having $n_{\mathrm{co}}=3.41, n_{\mathrm{cl}}=$ 1.5 , width $w=1.17 \mu \mathrm{m}$, and very small core thickness $t=$ $180 \mathrm{~nm}$. Our choice is motivated by the use of high index contrast waveguides in existing photonic devices involving PPC or TW structures. The length was chosen to be sufficiently large, $l=21 \mu \mathrm{m}$, to check the precision in the single-mode excitation at the far end of the waveguide. We have examined the dynamical propagation of the first three $E^{y}$ modes, generated with the appropriate initial profiles. The investigated spectral range is sufficiently above the cutoff frequency of the third eigenmode (to ensure accurate computation of the propagation constant and mode profile with the mode-solver). Using the effective-index method, we have calculated this frequency to be $f_{c 3} \cong 142 \mathrm{THz}$. For excitation, we used Gaussian pulses modulating a sinusoidal carrier of frequency $f_{0}=193.5 \mathrm{THz}$, which coincided with the reference frequency in the nonstandard difference operators. The computed values of $\zeta_{i}$ in this study were: $\zeta_{1}=0.7093$, $\zeta_{2}=0.00449$, and $\zeta_{3}=0.2458$.

Fig. 3 shows the extracted propagation constants over the bandwidth of the corresponding excitation pulse, which were obtained by dividing the FFTs of the pulse's time history at two fixed observation points along the core [6, Ch. 15-16]. Note the excellent agreement between the values predicted by the NS-FDTD and the fully vectorial mode solvers for all three eigenmodes that confirms the precision of their excitation. 


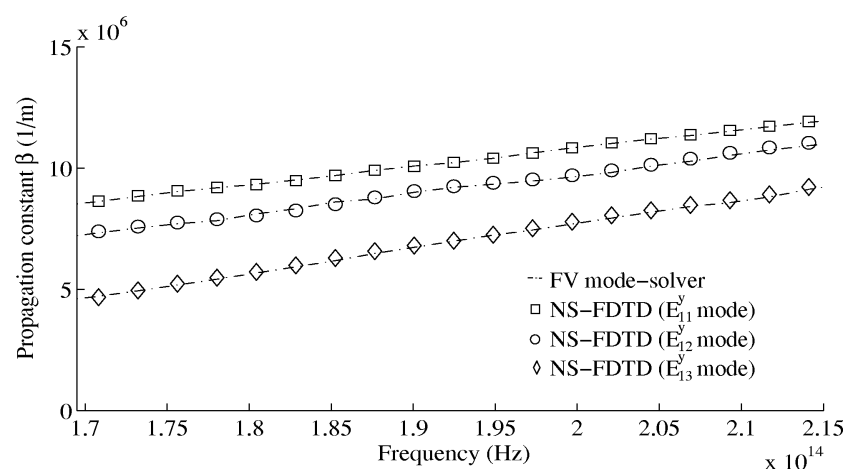

Fig. 3. Longitudinal propagation constants of the first three $E^{y}$ eigenmodes: Comparison between fully vectorial mode-solver (dashed line) and NS-FDTD (symbols).

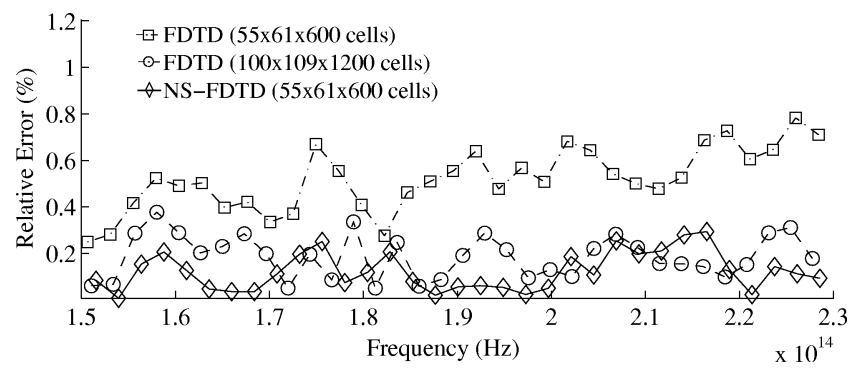

(a)

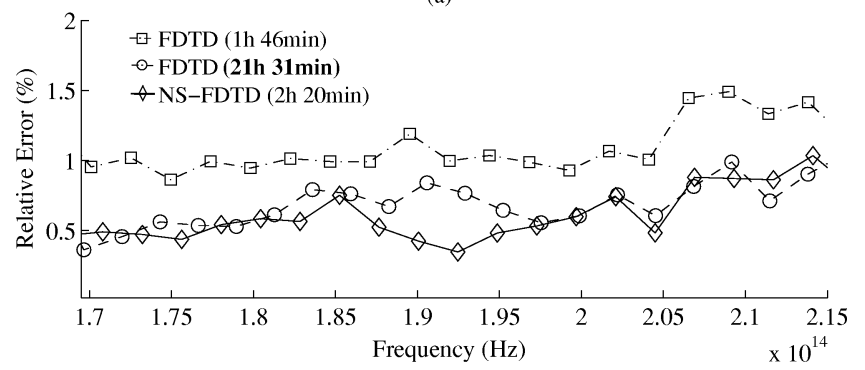

(b)

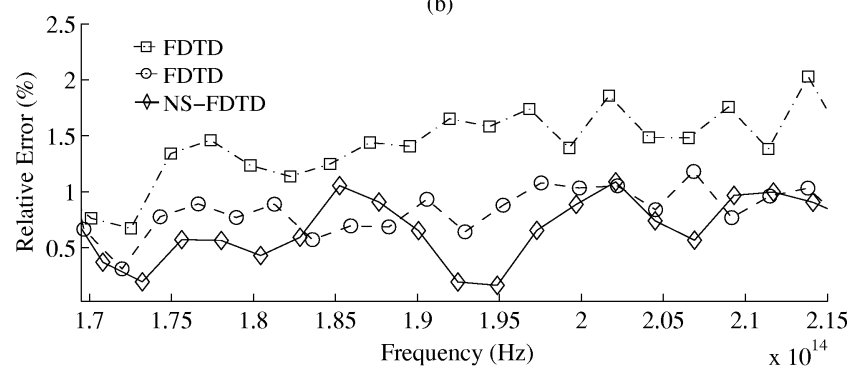

(c)

Fig. 4. (a) Relative error in the NS-FDTD and FDTD-computed propagation constant of the fundamental. $E_{11}^{y}$ eigenmode. (b) The same calculations as in (a), but for $E_{12}^{y}$ the eigenmode. (c) The corresponding calculations for $E_{13}^{y}$ the mode supported by the same structure as in both previous cases.
Fig. 4(a)-(c) illustrates the relative error, with respect to the mode-solver, of the conventional and NS-FDTD. In each case, it is found that the classical Yee scheme only attains the same levels of accuracy, especially in the high-frequency range, when the number of spatial grid points is increased by a factor of 6.5 with a corresponding significant increase in the computational time, as expected. The increase in the computational time for the NS-FDTD, however, is small due to the use of a $30 \%$ larger time-step than the maximum allowed one in the standard algorithm [4]. It is also verified that optimum performance for this form of the NS-FDTD is achieved within a narrow region around $f_{0}$ [9], where it is always found to be more accurate than the usual Yee formulation.

\section{CONCLUSION}

An accurate methodology, utilizing a fully vectorial excitation scheme, has been presented for the systematic analysis of arbitrary planar heterostructures with the 3D-FDTD method. Owing to the considerable reduction of the numerical dispersion and anisotropy errors, significantly enhanced computational performance has been demonstrated when the NS-FDTD formulation is used.

\section{REFERENCES}

[1] I. El-Kady, M. M. Sigalas, R. Biswas, and K. M. Ho, "Dielectric waveguides in two-dimensional photonic bandgap materials," J. Lightw. Technol., vol. 17, no. 11, pp. 2042-2049, Nov. 1999.

[2] K. L. Tsakmakidis, L. Gomez-Rojas, I. D. Robertson, O. Hess, P. A. Houston, and B. Weiss, "FDTD modeling of velocity mismatch in traveling-wave heterojunction phototransistor," Electron. Lett., vol. 40, pp. 452-454, Apr. 2004.

[3] S. T. Chu, S. K. Chaudhuri, and W. P. Huang, "Analysis of optical guided-wave devices with the finite-difference time-domain method," in Int. Symp. IEEE Antennas Propagat. Soc., vol. 1, Jul. 1992, pp. $257-260$.

[4] J. B. Cole, "High-accuracy Yee algorithm based on nonstandard finite differences: New developments and verifications," IEEE Trans. Antennas Propagat., vol. 50, no. 9, pp. 1185-1191, Sep. 2002.

[5] T. Kashiwa, H. Kudo, Y. Sendo, T. Ohtani, and Y. Kanai, "The phase velocity error and stability condition of the three-dimensional nonstandard FDTD method," IEEE Trans. Magn., pt. 1, vol. 38, no. 2, pp. 661-664, Mar. 2002.

[6] A. Taflove and S. C. Hagness, Computational Electrodynamics: The Finite-Difference Time-Domain Method, 2nd ed. Boston, MA, London, U.K.: Artech House, 2000.

[7] A. S. Sudbo, "Why are accurate computations of mode fields in rectangular dielectric waveguides difficult," J. Lightw. Technol., vol. 10, no. 4, pp. 418-419, Apr. 1992.

[8] C. L. Xu, W. P. Huang, M. S. Stern, and S. K. Chaudhuri, "Full-vectorial mode calculations by finite difference method," in Proc. Optoelectron., Inst. Elec. Eng., vol. 141, 1994, pp. 281-286.

[9] K. L. Shlager and J. B. Schneider, "Comparison of the dispersion properties of several low-dispersion finite-difference time-domain algorithms," IEEE Trans. Antennas Propagat., vol. 51, no. 3, pp. 642-653, Mar. 2003. 